\title{
CONOCIMIENTO Y MANEJO DE LOS INSECTOS Y PLAGAS DE LOS FRUTALES DE LA AMAZONIA ( $\left.{ }^{*}\right)$
}

Guy Couturier ${ }^{* *}$

\section{RESUMEN}

Un inventario de las plagas de los frutales se está realizando en la A mazonía Brasilera (M anaus) y peruana (I quitos). En este trabajo se presentan algunos ejemplos de plagas desconocidas, a veces especies nuevas para la ciencia, y del complejo parasitario. $\mathrm{H}$ an sido estudiadas algunas plantas de cultivo antiguo, la piña (Anunas comosus L.)

Bromeliaceae), la guanabana (Annona muricata L. Annonaceae), los cítricos (Citrus sp. Rutaceae); pero sobre todo los de cultivos recientes, tales como el camu camu (M yrciaria dubia H.B.K. Myrtaceae), elarazá, (Eugenia stipitata MC Vaugh M yrtaceae), y la cocona(Soltinum sessiliflorum Dunal Solanaceae).

Se ha demostrado que, en los cultivos clásicos existen, además de las plagas básicas, plagas anteriormente desconocidas, adaptadas a partir del medio natural. 'odas las plagas de los cultivos nuevos constituyen problemas actuales, nuevos o potenciales.

Palabras claves: insectos, plagas, frutales, A mazonía, Perú, Brasil.

\section{INTRODUCCION}

Las consecuencias de las importantes modificaciones que afectan la amazonía son numerosas, y numerosos son los políticos, ecólogos, agrónomos que se preocupan e una planificación racional, y de la valorización de las tierras vírgenes.

Entre los diferentes factores de éxito o fracaso que se presentan en el marco de una operación de desarrollo agrícola, se encuentran los problemas fitosanitarios; estos problemas son particularmente agudos cuando se trata de nuevos cultivos cuyas plagas con desconocidas.

Desde 1987, se vienen desarrollando inventarios de los insectos plagas de los frutales en las zonas de Iquitos y $M$ anaus; especialmente en los nuevos cultivos tales orno las M yrtaceas Eugenia stipitata, M yrciaria dubia y la palmera Bactris gasipaes, así

Comunicación presentada en el ler. Simposium Internacional de Ecología Tropical A mazónica, 3-8 noviembre de 1991, Iquitos - Perú

Investigador QRSTOM, 213 rue Lafayette, 75480 Paris cedex 10, Francia. Dirección actual ORSTOM. A partado 18-1209 Lima 18 Perú. 
como e los cultivos clásicos como los cítricos Citrus spp. Y la Bromeliacea Ananas comosus. En este estudio presentamos algunos casos significativos de plagas nuevas o no reportadas en la literatura antes de estos estudios, así como al gunos casos de complejos parasitario incluyendo plagas bien conocidas.

\section{LOCALIZACION Y METODOS}

Los inventarios y las observaciones han sido realizados en diferentes tipos de cultivos representativos tales como:

En la amazonía Brasilera, en la región de Manaus, en plantaciones privadas, huertos familiares y plantaciones experimentales del Instituto Nacional de Pesquisas de la A mazonía (INPA) ubicadas en zonas de "tierra firme" (carretera M anaus-Boa vista y $M$ anaus-I tacoatiará), en "varzea" en las islas C areiro frente a $M$ anaus.

En la amazonía Peruana, región de lquitos, en plantaciones privadas y experimentales de los siguientes Institutos: Instituto de Investigaciones de la A mazonía peruana IIAP), Instituto Nacional de Investigación A graria y Agroindustrial (INIAA) e Instituto Veterinario de Investigaciones del trópico y de altura (IVITA). Estas plantaciones se ubican en terrenos de altura "tierra firme" (carretera Iquitos-Nauta y Jenaro Herrera) yen restinga "varzea" (Padre isla e isla M uyuy)

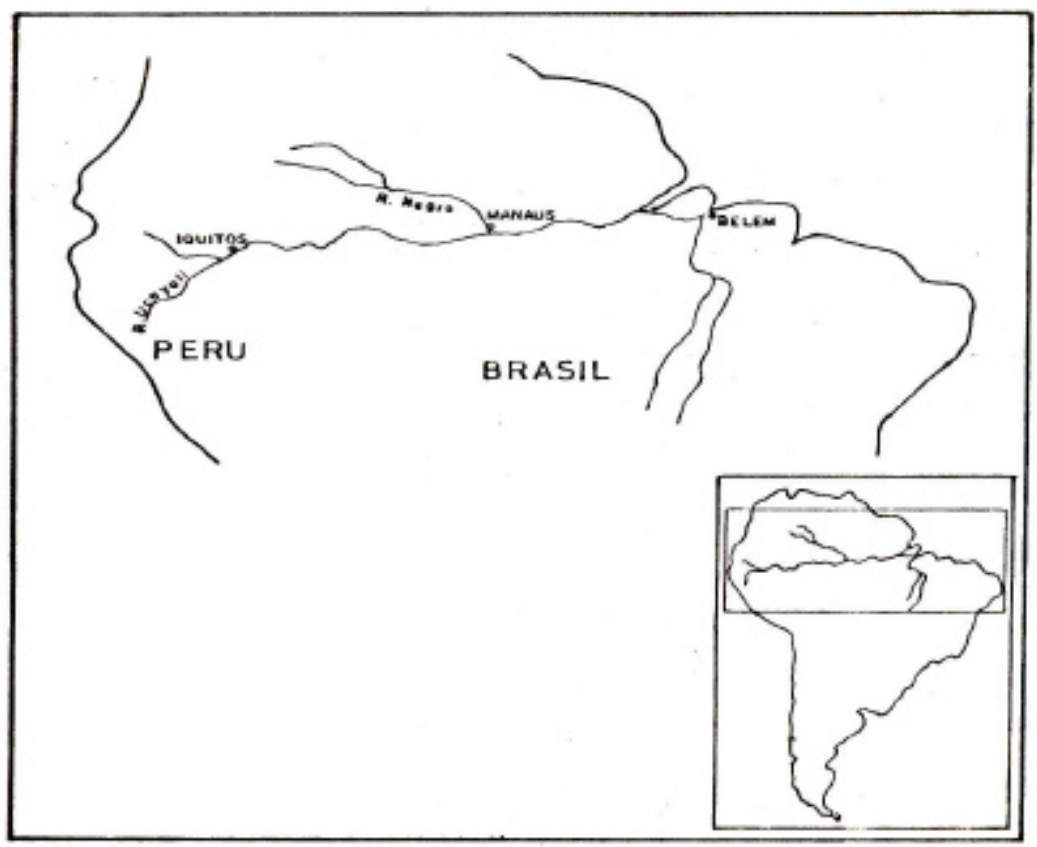

FIGURA 1. Lugares de Muestreo en Brasil y Perú 
Las muestras han sido colectadas visualmente, sin uso de trampas, muestreándose frutos, hojas y ramas; las larvas o ninfas obtenidas han sido criadas en la planta hospedera de origen, cuando han sido necesario la obtención de adultos para la Identificación. Las identificaciones han sido realizadas por taxónomos especialistas para cada grupo taxonómico obtenido.

\section{RESULTADOS}

\subsection{Plagas nuevas o no reportadas en los cultivos antiguos}

Ananas comosus (Bromeliaceae), piña. En las localidades prospectadas, pocas son las especies de insectos que atacan el cultivo de la piña. Entre estos el heteroptero Coreidae Thlastocoris laetus M ayr, se señala por primera vez como plaga; la especie es frecuente en la región de Jenaro Herrera, en el Perú yen el $N$ orte de $M$ anaus, en el Brasil, ha sido, también, encontrada en poblaciones importantes sobre piña nativa en la misma zona de M anaus. L as plantas que albergan más de 20 individuos se amarillean, secan y mueren.

Citrus spp. (Rutaceae) cítricos. En las plantaciones ubicadas al Norte de $M$ anaus, ha aparecido una nueva plaga desde 1987, el lepidoptero Cossidae L angsdorfia sp.; las orugas comen la parte viva de la corteza y son limitadas a la parte baja del tronco; los daños que ocasionan son importantes. El $98 \%$ de las plantas de Citrus reticulata (mandarina) están atacadas (Couturier y Rezende do V alle, en preparación). Esta es una especie nueva para la ciencia, en curso de estudio por J.P. Donahue (L os A ngeles USA).

Annona muricata (A nnonaceae), guanábana. Este frutal muy cultivado en la región de Manaus, da lugar a transacciones comerciales importantes. Es atacado por varias plagas (Hamada et al 1991); entre estas una especie recientemente descrita el homoptero $M$ embracidae Membracis suctifructus (Boulard \& Couturier, 1991), causa muchos daños, ocasionando el desecamiento de las flores y de los frutos jovenes.

\subsection{Plagas de los nuevos cultivos}

Consideramos como cultivo nuevo, las especies nativas cultivadas desde hace menos de 20 años en las áreas consideradas; ya sea en plantaciones experimentales 0 privadas, con fines comerciales. Ninguna plaga había sido señalada antes de estos estudios.

Myrciaria dubia (M yrtaceae), camu-camu. Este frutal es un arbusto que crece, naturalmente, en las riberas de los ríos y lagos de la cuenca amazónica; esta cultivado experimental mente en los campos del IIAP e 
INIAA en Iquitos yen el INPA en Manaus. Algunas plantaciones privadas existen en Iquitos desde hace poco.

Sus plagas han sido estudiadas por Couturier et al (1992, en prensa); 42 especies de fitófagos han sido citadas. Las mas importantes, que afectan la productividad son: un homoptero Psyllidae Tuthillia cognata, descrito por Hodkinson et al en 1986 sobre tres ejemplares de una planta hospedera desconocida. Al año siguiente se descubre la especie como plaga importante del camu camu en Jenaro Herrera y $M$ anaus (Burckhardt \& Couturier, 1988); el insecto, que se encuentra en todas las plantaciones, provoca el desecamiento de las hojas apicales impidiendo el desarrollo de las ramas. Un coleoptero Scolytidae Xylosandrus compactus Eichhoff, que ataca los plantones en vivero causando la pérdida del $40 \%$ de la producción (Couturier \& Tanchiva, en prensa). Otro coleoptero Curculionidae Conotrachelus sp., se desarrolla en las semillas, provocando la pudrición de los frutos; ésta plaga esta invadiendo poco a poco las plantaciones de Iquitos.

Diversas especies de queresas representan, también, un peligro para el cultivo de camu-camu y están en curso de estudio. Los otros fitofagos encontrados deben de ser considerados como secundarios.

Eugenia stipitata (M yrtaceae), arazá. Es un frutal nativo de mucho potencial para la agroindustria; según un estudio de mercado, realizado en 1986 en el marco del programa de investigación en cultivos tropicales (INIAA), existe una demanda potencial de 180 a 240 toneladas de fruto en la ciudad de Iquitos. El arazá se encuentra en forma silvestre en el departamento de Loreto, especialmente en la provincia de Requena (Picón Baos, 1989). La biología floral y la ecología han sido estudiadas por Falcao et al (1988). Cultivos experimentales existen en el INIAA, el IIAP, el INPA. Algunas plantaciones privadas van desarrollándose. Desde hace poco, el fruto es procesado por el INIAA/IIAP (U niversidad Nacional de la A mazonía Peruana).

L as plagas del arazá están en curso de estudio, hasta ahora se considera que, en la zona de Iquitos - J enaro Herrera, cuatro plagas constituyen actualmente, un factor limitante en cuanto a la calidad y la cantidad de frutos producidos.

Las larvas de dos especies de picudos (Coleoptera Curculionidae) se desarrollan en los frutos, atacando las semillas y la pulpa; su repartición es muy heterogénea entre las diferentes plantaciones estudiadas. Dípteros de la familia Tephritidae, Anastrepha sp, plaga bien conocida en varios frutos de los neotrópicos, así como de la familia Lonchaeidae, de menor importancia, se desarrollan en la pulpa de los frutos (Picón Baos, doc. cit., señala la presencia de Anastrepha 
striata) su repartición también es muy heterogénea según las plantaciones. Una tercera especie de Curculionidae se alimenta, a la estadía adulto, de las hojas tiernas y de los botones florales; sus daños pueden ser muy importantes. Otras plagas, tales como Lepidópteros Mimallonidae, Psychidae, Pyralidae, Megalopygidae, son de interés secundario. El arazá al contrario del Camu camu, está muy poco atacado por las queresas. Solanum sessiliflorum (Solanaceae), Cocona. La Cocona es un fruto muy frecuente en los mercados de Iquitos, Manaus, y otras ciudades de la A mazonía. La ecología, el cultivo y la composición química del fruto han sido estudiadas por Pahlen (1977). Existen cultivos experimentales en el INPA en Manaus yen el INIAA en Iquitos. En Manaus se han registradas 14 especies de insectos fitófagos (Couturier, 1988), (Tabla 1).

La mayor parte de estas especies son conocidas en otras solanáceas cultivadas. El heteroptero Tingidae Corythaica cyathicollis Costa, por la importancia de sus daños en las hojas, justifica intervenciones fitosanitarias. Esta última especie se encuentra en Iquitos, así como el chinche Edtessa rufomarginata De Geer, y un coleoptero Meloidae Lytta sp.

TABLA 1 : Insectos observados en la cocona (Solanun sessiliflorum Dunal) en la plantación experimental del INPA en Manaus (según Couturier, 1988).

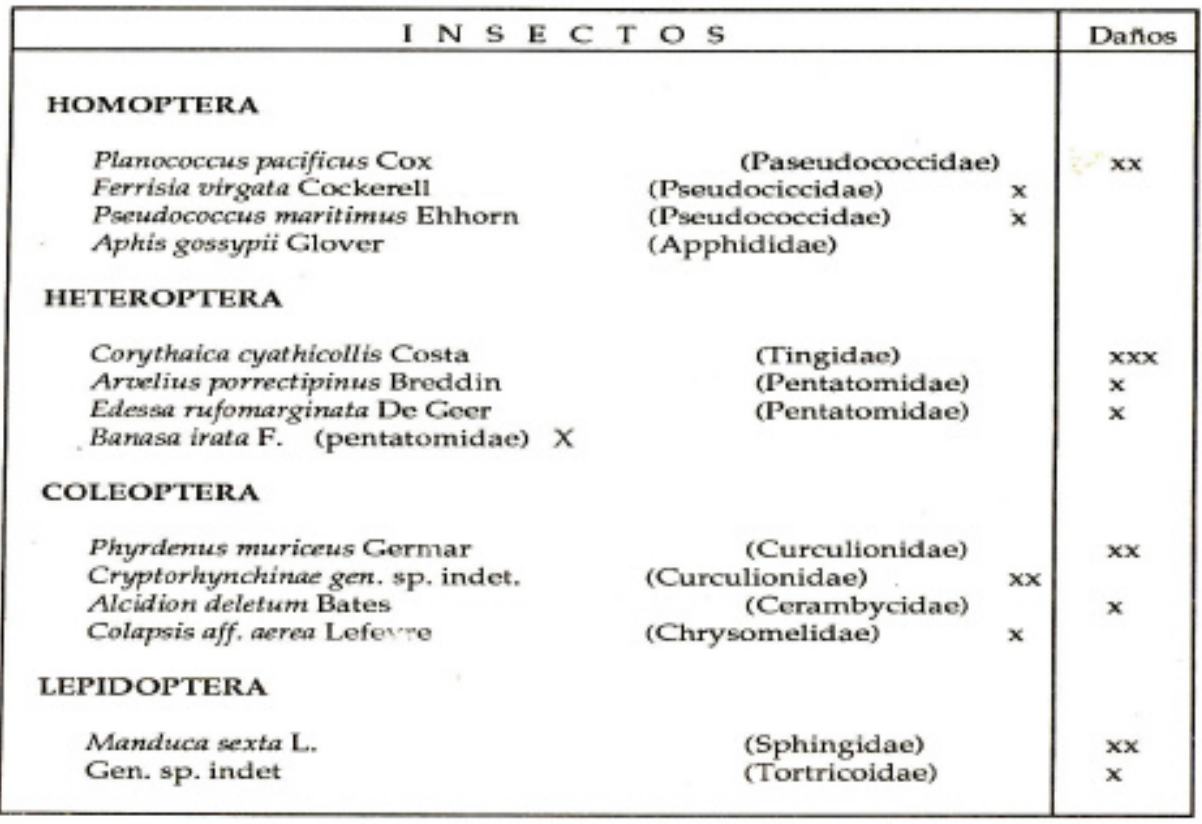


el que, por su abundancia, puede defoliar totalmente la planta (observaciones realizadas por el autor $X, R$. Cardenas Mori y $E$. Tanchiva Flores, en la estación el Dorado del INIAA, datos no publicados).

B actrís gasipaes (Palmae), pijuayo. El número de plantaciones, con fines de selección y. mejoramiento así como para la producción comercial, está creciendo en varios países amazónicos y de A mérica Central. El al to potencial económico del pijuayo (Producción de frutos y de palmito) ha dado lugar a numerosos estudios en varios países amazónicos; entre los cuales podemos citar los trabajos de Clement (1988), Clement \& Mora Urpi (1987) y Mora Urpi (1983).

Se considera que el pijuayo tiene una buena resistencia a las plagas (Pinedo, 1989); sin embargo existen; estudios realizados en Colombia (Pava et al., 1981) demuestran que son, en su mayoría, plagas conocidas de la palma aceitera y del cocotero. En el Brasil, en las plantaciones del INPA en Manaus, una nueva plaga: el chinche coreidae Leptoglossus lonchoides, has idoestudiado por Couturier et al. (1991). La planta hospedera de este insecto era antes desconocida; se trata de una adaptación muy reciente al cultivo. L. Ionchoides presenta poblaciones importantes y contribuye a la caída de los frutos jóvenes. En el estado de Bahia en Brasil, Lourencao \& Bovi (1987) reportan cuatro especies de insectos que dañan, ya sea la inflorescencia, o la pulpa del fruto.

\section{DISCUSION}

Se ha demostrado, a partir de algunos ejemplos, que los insectos plagas de los cultivos frutales, en las regiones amazónicas estudiadas, son poco conocidas. El desarrollo creciente de las áreas cultivadas, la introducción en los sistemas de producción de nuevos frutales favorece el incremento de las plagas, ya sean clásicas 0 nuevas. La escasez de informaciones, tanto en la A mazonía brasileña (Ronchileles \& Hamada, 1991), como en la A mazonía peruana es muy preocupante.

En efecto, paradójicamente, la desaparición progresiva del bosque amazónico atrae la atención del mundo entero; se habla de protección del medio natural, de estudio y de conservación de la biodiversidad, pero falta información básica sobre las plagas de los cultivos y de estudios sobre su manejo. La falta de conocimientos o de medios adecuados de manejo, trae como consecuencia muchas pérdidas de tierras vírgenes; a causa del bajo rendimiento debido, entre otros factores, a los insectos. Se pueden citar muchos ejemplos de tierras mal manejadas y que son abandonadas por causa de plagas, ya sean insectos o enfermedades. El desarrollo de la A mazonía solo será posible si se logra un mejor rendimiento de las tierras (Villachica et al., 1990). Por otra parte, la producción con fines de exportación requiere de productos de alta calidad, sin rastros de daños, ni rastros de pesticidas, de modo que, el control de las plagas, tiene un rol muy importante en el desarrollo y la preservación de la Amazonía. Las alternativas son 
numerosas, pero de difícil aplicación; no obstante, el Perú tiene una gran experiencia en los métodos de control integrado (A guilar, 1980; Beingolea, 1980,

1990; Cisneros, 1989; Lamas, 1980) que debe de difundirse entre los agricultores y productores amazónicos.

Sin embargo se requieren de más estudios básicos, particularmente cuando se trata de nuevos cultivos promisorios. En efecto para poder controlar efizcamente los insectos, es necesario conocer su identidad precisa, su biología, y entender las complejas interacciones entre ellos y sus plantas hospederas, así como las prácticas agrícolas que permitan reducir el nivel de los daños.

En caso de desarrollo importante de cualquier cultivo, sean monocultivos, sistemas agroforestales o pequeñas plantaciones familiares, se deberán proponer alternativas ecológicamente satisfactorias para el control de estas plagas en el marco del concepto de "Control Integrado".

\section{AGRADECIMIENTO}

El autor agradece al Dr. Kember Mejia (IIAP, Iquitos) quien ha revisado el texto en español.

\section{BIBLIOGRAFIA}

A GUILAR, P.D. 1980. A puntes sobre el control biológico y el control integrado de las plagas agrícolas en el Perú. Rey. per. Ent. 23 (1): 83-110.

BEINGOLF A 0.1980. El futuro del control integrado de las plagas agricolas (C.I.P). Rey. per. Lnt. 23,(1): 7-15.

BEINGOLEA, G., D. OSCAR. 1990 (1991). Sinopsis sobre el control biológico de plagas insectiles en el Perú, 1909 - 1990. Rey. per. Ent. 33: 105-112.

BOULARD, M. \& G. COUTURIER, 1991. Membracis fructisuctus n. sp., nouvean cicadaire nuisible aux plantes fruitiers Amazoniennes (Homoptera, M embracoidea, M embracidae). EPME, Trvx Lab. Biol. Evol. ms. 4: 55-60

BURCKHARDT,D.\& . COUTURIER, 1988. Biologyand taxonomy of Tuthillia cognata (Homoptera Psyltoidea, a pest of Myrciaria dubia (M yrtaceae). Annls. Soc. ent. Fr. (NS.), 24 (3) : 257 - 261.

CHAVEZ FLORES, W. B. NODA, \& C.R. CLEMENT, 1990. Genetic/phenotypic studies on spines in pejibaye (Bactris gasipaes H. B. K., Palmae). Rey. Brasil. Genet. 13 (2): 305 -312. 
CISNEROS, F.M. -1989. Manejo integrado de plagas en el Perú y el mundo. A groenfoque, Lima, № 33: 26-27.

CLEMENT, C. R. - 1988. Domestication of the pejibaye palm (Bactris gasipaes): past and present. Adv. Econ. Botany, 6:155 - 174.

CLEMENT, C. R. \&J. MORA URPI, 1987. The pejibaye (Bactris gasipaes HBK, A recaceae):

multi use potencial for the lowkmd humid tropics. Econ. Botany, 41: 302-311.

COUTURIER, G. 1988. Alguns insetos depredadores do Cubiu (Solanum sessiliflorum var. sessiliflorum Dunal, Solanaceae) na regiao de Manaus Am. Acta A mazónica, 18 (3-4): 93-103.

COUTURIER G., C.R. CLEMENT \& P. VIANA FILHO 1991. Leptolossus Ionchoides (Heteroptera, Coreidae), agente de la caída de los frutos de Bactris gasipaes (Palmae) en la A mazonia central. Turrialba, 41(3), en prensa.

COUTURIER, G.; H. INGA SANCHEZ, H. \& E. TANCHIVA FLORES, 1992. LOS Insectos fitófagos que viven en Myrciaria dubia H.B.K, Myrtaceae, frutal cultivado en la A mazonía Peruana, Folia A mazónica, 3, en prensa.

COUTURIER, G. \& E. TANCHIVA FLORES, Xylosandrus compactus, nueva plaga del camu camu (M yrciaria dubia H.B.K., M yrtaceae) en la región de Iquitos, A mazonía Peruana, Bol. Fit. FA O, en prensa.

COUTURIER, C. $\&$ J. REZENDE DO VALLE, Langsdorfia sp. (Lepidoptera cossidae Hypoptinae), nouveau ravageur des Citrus dans la región de $M$ anaus (Brasil, A mazonas), en preparation.

FALCAO, M. A., W. B., CHAVEZ FLORES, S.A. FERREIRA, N., C. R., CLEMENT, M. J.B. BARROS, J. M. C. BRITO. De \& 1. C. 1. SANTOS doS - 1988. Aspectos fenológicos y ecológicos do arazá boj (Eugenia stipitata M C Vaugh) na A mazonia central. I plantas juvenis. A cta A mazónica, 18 (34): 2738.

HAMADA, N., G. COUTURIER, et B. RONCHI TELES, 1991. Insectos associados a gravioleira (Annona muricata) na Amazonía Central. XIII eme Congresso brasileiro de Entomologia, $20-25$ J aneiro 1991, Recife. 
HODKINSON 1. D., R. G. BROWN, \& D. BURCKHARDT. 1985. Tuthillia, a new tropical psyllid genus (Homoptera Psylloidea). Syst. Ent., 11: 53 - 60.

LAMAS, J. 1980. Control de los insectos-plaga del algodonero en el Perú -Esquema de la planificación de una campaña de control integrado y su problemática. Rey. ent. Per. 23, (1): 1-6.

LOURENÇAO, A. L. \& M.L.A. BOVI. 1987. Insectos nocivos apupunheira (Bactris gasipaes H. B. K.). XI e Congresso brasileiro de entomologia, 12-17 jul. 1987, campinas Resumos, vol 1: 62.

MORA URPI,J. 1983. El pejibaye (Bactris gasipaes H.B.K), origen biología floral y manejo agronómico. In: Palmeras poco utilizadas de América Tropical. FA 0/CATIE. Turrialba: 118- 160.

PAHLEN, A. von der 1977. Cubiu (Solanum topiro Humb \& Bonpl.), urna frutifeira da A mazonia. A cta amazonica, 7 (3): 301 - 307.

PAVA, J. O.E., CASTILLO, \& A. GONZALES. 1981. Consideraciones ecológicas preliminares sobre aspectos de interés fitosanitarios de la palma de chontaduro Bactris gasipaes H.B.K., en algunas regiones de los departamentos del valle y choco. Thesis, Palmira Colombia, 175p. anex.

PICON BAOS, C. 1989. Cultivo de arazá, manual técnico N2 2, serie didáctica, INIAA, Iquitos - 7p.

PINEDO, M. P. 1989. Técnicas para la producción de palmito y fruta de pijuayo (Bactris gas q.' aes H. B. K.). Informe Técnico N 2 5, INIAA, Iquitos, 11 p.

RONCHITELES, B. \& . N HAMADA, 1991. Estudios Biológicos de insectos prejudiciais a agricultura como subsidio para o manejo de pragas na amazonía. In 'bases científicas para estrategias de preservazo edesenvolvimento da amazonía: Fatos e perspectivas", IN PA, M anaus: 233-236.

VILLACHICA, H., J.E. SILVA, J.R. PERES \&. C.M.C: ROCHA da 1990. Sustainable agricultural systems in the humid tropics south America, in: Sustainableagricultural systems, 5. W. Conservation Institute, Ankeny: 391 437. 